\title{
Travel Experience with Collective Intelligence
}

\author{
Young Hoon $\mathrm{Oh}^{1}$, Da Young $\mathrm{Ju}^{2}$ \\ ${ }^{1}$ School of Integrated Technology, Yonsei University, Incheon, Korea \\ ${ }^{2}$ Yonsei Institute of Convergence Technology, Yonsei University, Incheon, Korea \\ 50hoon@yonsei.ac.kr1',dyju@yonsei.ac.kr²
}

\begin{abstract}
Smartphone has become a requisite for everyday life. We use it for checking e-mails, calling friends, and surfing mobile applications. Traveling with mobile phone is no exception. But existing media such as travel guidebook, blog, or current mobile applications are too difficult to organize information readily. Therefore, we propose the Collective Intelligence System (CIS) which resolves the inconvenience of travelers. The system is formed with the empirical knowledge of 'Local' and the check-in log of Social Network Service (SNS). As the system regulates certain reward for the tips, constituents are able to give helpful advice spontaneously. Moreover it can be applied to mobile application that the system provides usercentered travel route as well as share travel experience. We present Travel Preference Analysis (TPA) that analyzes the tendency of each user. It checks overall response to specific information when it is shown up on the mobile application. For example as user delete a destination among the given route, the application checks its character and try to figure out why it is excluded. Then TPA transfers the preference to the CIS. Therefore, the more users edit information, the better system improves the result. Our future work will explore more precise factors to provide user-centered travel experience.
\end{abstract}

Keywords: Travel route, Collective Intelligence, Information, User-centered, Preference, Experience

\section{Introduction}

Development of transportation and globalization has led people to enjoy much affluent leisure times. One of the memorable activities that people cherish is travel. Generally, the word 'travel' means that sightseeing, shopping, or eating traditional foods. But travel 'somewhere' means that people go places without enough information and reliable information for travelers is essentially required. The best information is advice of people who have a lot of experience at certain place. Therefore, we propose Collective Intelligence System (CIS), which helps to generate user-centered travel route and share helpful advice. Based on the mobile application, the system consists of priori knowledge [1] and check-in log of Social Network Service (SNS) Friends. Moreover, we present Travel Preference Analysis (TPA), which is an effective measurement to read travel trend in a mobile device.

\section{Concept of Collective Intelligence System}

Information merely focused on quantity is spoilt for choice. It is obvious that travelers are absolutely lacking in practical, empirical information. Thus, we present CIS to provide affluent travel experience. It is applied to mobile application that generates travel route automatically. The system shares helpful advices for people who need credible travel information.

The constituent of CIS is classified into two categories One is 'Local' who lives at the place where tourists are interested in. There are various kinds of people abroad, for instance, people who run their own business, international students, or immigrant. The other member is SNS Friends, linked with personal account. 'Friends' indicate the concept of 'Friend' of Facebook, and 'following' user of Twitter can be included as well.

Basically, all members ought to provide neutral information. They are not allowed to share information related with own business, similar categories of business or job. However, you can lead a horse to water, but you cannot make him drink. Therefore, we motivate constituents by rewarding promised forms of incentives to shape collective intelligence. The form can be diversely proposed. One of the examples is reducing advertisement charges for people who run own company abroad. At the same time, the incentive can be varied depends on their activity. That is, the more they participate in forming intelligence, the more they get rewards.

\section{Mobile application based Crowdsourcing}

There are plenty of unpremeditated cases that tourists face up with. In case of state occasion, several avenues are blocked, people are regulated to move through the way, and they are under the necessity of changing schedule after all. In this situation, previous media, which communicates information in one-sided way, cannot suggest physical solution. Even though the communication form of information has shifted to mobile environment, the media do not optimize interacting method for travelers. These are the following several problems of existing media. First of all, existing media cannot meet each one's travel tendency and demand. Secondly, especially book cannot reflect realtime information. Furthermore, share of information is not decided by user itself [2]. That is, any kind of 'user generated content' are not accepted directly. In the case 
of Internet, it is able to transfer and receive information instantaneously however the information is not provided as an optimized form for tourists. Thirdly, information for travelers is not integrated itself and scattered around. For example, if a traveler who hopes to travel Tokyo, he/she has to 'find' information where it is. It means that he/she has to search where to go, how to move, or what to do. That's the reason why people depend on the plan of travel agencies. Thus, the method of communication should be changed.

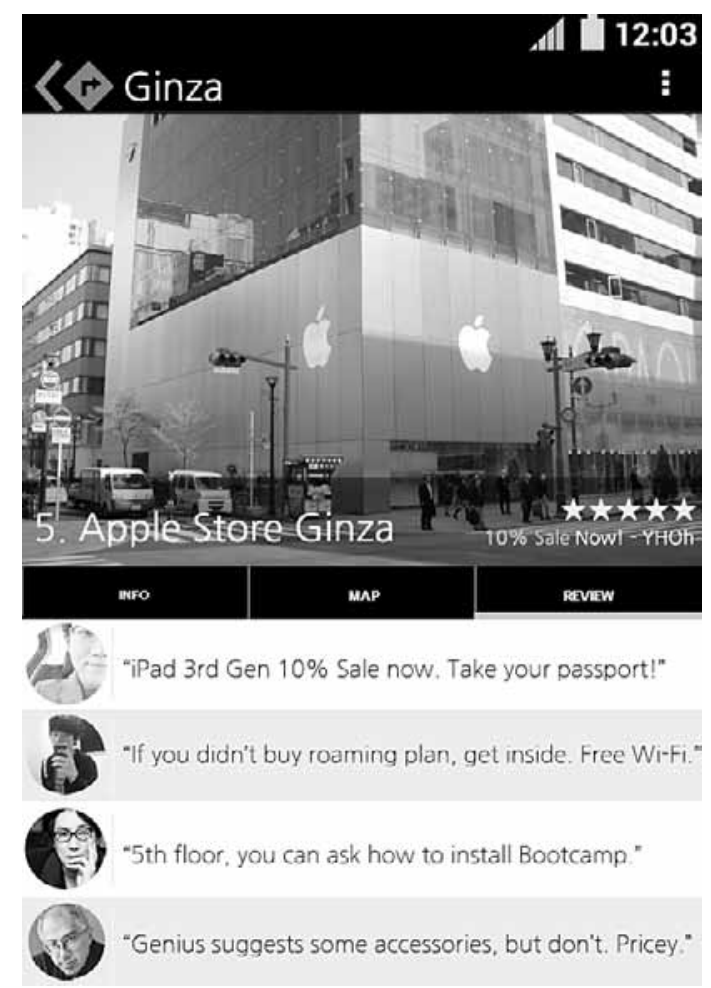

Figure 1. Example of collective intelligence

The CIS is the system that collects information from mobile application. The biggest strong point of mobile based crowdsourcing is instantaneous interaction. Think how you feel when you meet an old friend abroad by chance. You may feel relaxed and even you may ask where to travel around. Talking with Local who knows well facilitate strangers to get helpful information [3]. Moreover, it is much easy to ask share. As a result, collective Intelligence can be formed quickly. Secondly, the feasibility of sharing information is important because it is nothing but creating noiseless shout. If nobody hears, nobody speaks. Thirdly, everybody has expertise in around my home. It can be an easy opportunity to give routine advice to others. For example, if the navigation system calculates and shows for the shortest path is A to B, most of people take it up as it is. However, people who have empirically experienced themselves are able to instruct alternative, A to C. Likewise, whether tips given by local exists or not prevent tourists from making a vain effort. Lastly, the most important thing is the quality. Constituents must provide reliable information, and it is conformed to objective opinion. As the members are not allowed to provide information related with them, the intelligence becomes more helpful than previous media. Moreover, the information is consistently verified by others including Local, and it finally becomes intelligence.

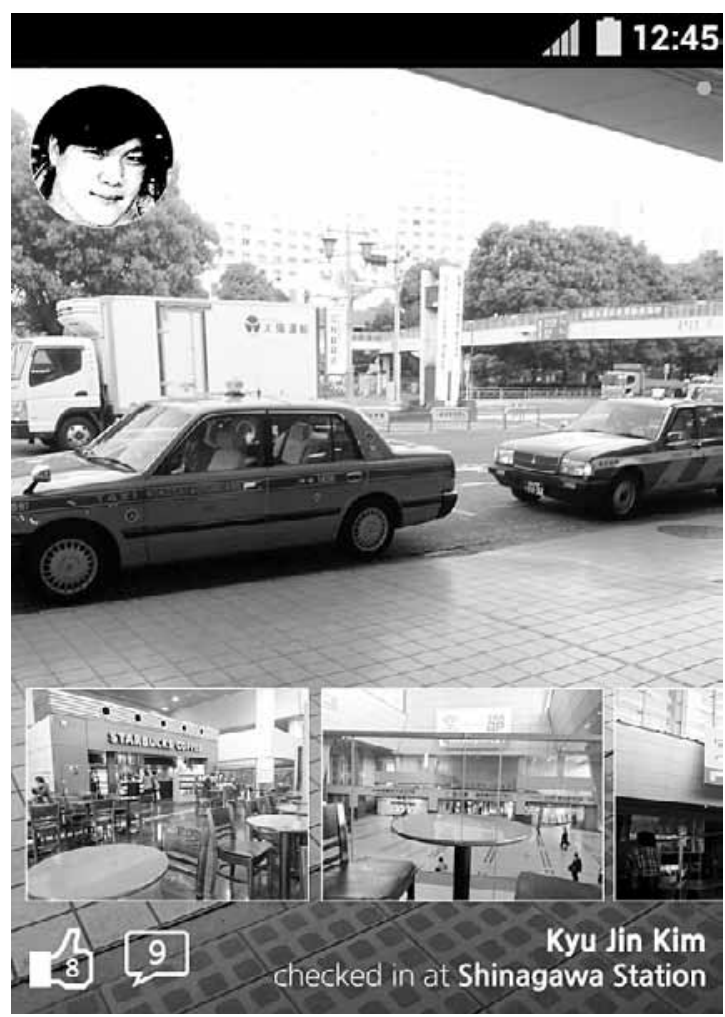

Figure 2. Example of Social Network Check-in

How about the information on SNS? We do not throw all newsfeeds from SNS. In other words, among thousands of tweets, posts, or messages, checked in place where you are interested in is selectively provided. It is the most reliable way to get information since the check-in log is the token that user had come by there. Additionally, SNS is built as a platform for share own activities, the motive of asking advice is natural. Therefore, the collective intelligence using location based SNS data makes people to get useful travel routes as well as establish a foothold to increase interaction [4, $5,6]$.

\section{Travel Preference Analysis}

The reason why some people are not willing to go packaged tour is easy. However, it cannot reflect every different person's preference. Although the technology of conveying information has been drastically developed, there are no means to grasp future action and tendency of multiple informavores immediately. As a result, it has been left as a long-cherished desire to previous media. Therefore, we present the Travel Preference Analysis to figure out what they like.

Unlike previous media platforms, mobile device is a favorable condition to check action of users. TPA analyzes the instant reaction on mobile application when it shows diverse kinds of travel information. For instance, automatically generated travel route, check-in 
wall post, or daily issued place can be given. People may read, bookmark information, or delete certain places from the travel route. Once the event occurs, the mobile application checks the following characteristics of targeted destinations: Category, cost, travel time to destinations, and average time to travel around.

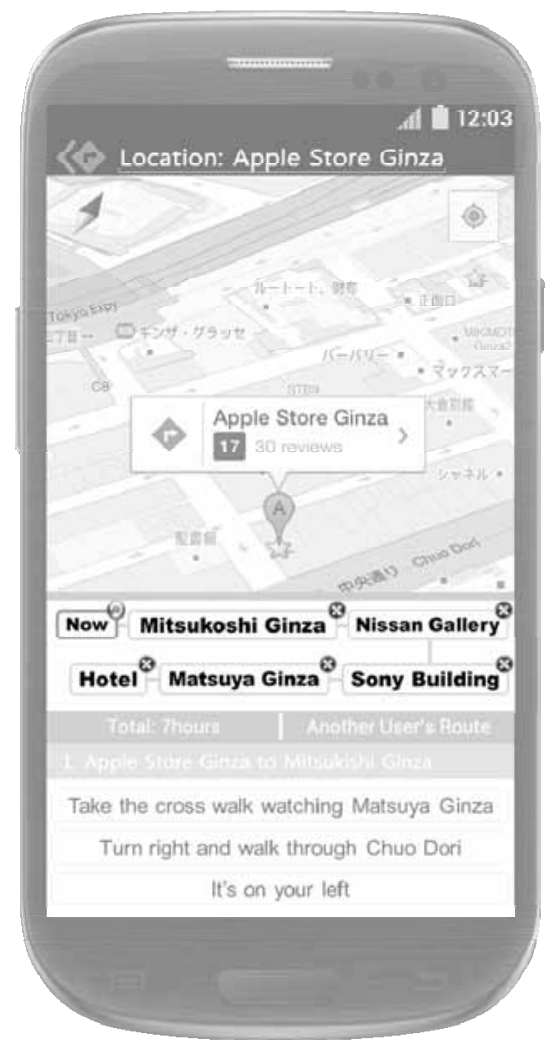

Figure 3. Deleting destinations from given travel route

For instance, if a certain shopping center is repetitively deleted, the system lowers the probability of compose 'museum' category. In another case, if the user browses traditional market and put it in the shopping cart, then the likelihood of route generation including it is weighted. Lastly the analyzed information is transferred to CIS since it can help users to get personalized travel information. Compared to survey and interview, TPA is highly efficient in productivity of analyzing personal trend. In conclusion, as the usage form of mobile application is accumulated, it can provide much personalized travel experience.

\section{Conclusion}

This research proposes Collective Intelligence System, which alleviates the inconvenience of travelers. Through this system, it provides personalized travel route that people had to plan themselves. Moreover, it distributes benefit to both members and travelers. Travel Preference Analysis researches the reaction in respect of given travel route and contributes to compose user-centered travel route automatically. In the future, we are going to explore more public components of collective intelligence and travel preference.

\section{Acknowledgement}

"This research was supported by the MKE(The Ministry of Knowledge Economy), Korea, under the "IT Consilience Creative Program" support program supervised by the NIPA(National IT Industry Promotion Agency)" (NIPA-2013-H0203-13-1002)

\section{References}

[1] Patel, K., Chen, M. Y., Smith, I., \& Landay, J. A.: Personalizing routes, proc. of the 19th annual ACM symposium on User interface software and technology, 187-190, 2006

[2] Gregg, D. G.: Designing for collective intelligence, Communications of the ACM, 53(4): 134-138, 2010

[3] Cho, E., Myers, S. A., \& Leskovec, J. : Friendship and mobility: user movement in location-based social networks. In Proceedings of the 17th ACM SIGKDD international conference on Knowledge discovery and data mining, 1082-1090, 2011

[4] Yu, Y., Kim, J., Shin, K., \& Jo, G. S. : Recommendation system using location-based ontology on wireless internet: An example of collective intelligence by using 'mashup' applications, Expert systems with applications, 36(9) : 11675-11681, 2009

[5] Sueda, K., Duh, H. B. L., \& Rekimot, J.: Social life logging: can we describe our own personal experience by using collective intelligence?, In Proceedings of the 10th asia pacific conference on Computer human interaction, 41-50, 2012

[6] Eagle, N., Pentland, A. S., \& Lazer, D.:Inferring friendship network structure by using mobile phone data, proc. of the National Academy of Sciences, 106(36): 15274-15278, 2009 